\title{
DIFERENCIAS ENTRE EMPLEABILIDAD, INSEGURIDAD LABORAL Y SALUD EN TRABAJADORES Y DESEMPLEADOS
}

\author{
DIFFERENCES BETWEEN EMPLOYABILITY, \\ JOB INSECURITY AND HEALTH IN ORKERS, \\ AND UNEMPLOYED
}

\author{
Miguel ANGel SerRano RosA ${ }^{1}$, SANDRA BAEnA $^{1}$ Y \\ FRANCISCO MOLINS-CORREA ${ }^{1}$
}

Cómo referenciar este artículo/How to reference this article:

Serrano Rosa, M. A., Baena, S. y Molins-Correa, F. (2018). Diferencias entre empleabilidad, inseguridad laboral y salud en trabajadores y desempleados [Differences between Employability, Job Insecurity and Health in Workers, and Unemployed]. Acción Psicológica, 15(1), 87-102. https://doi.org/10.5944/ap.15.1.20945

\section{Resumen}

El desempleo y la inseguridad laboral tienen efectos negativos sobre la salud, aunque la empleabilidad podría mediar dichos efectos. El presente artículo tiene como objetivo analizar las posibles diferencias entre trabajadores y desempleados en empleabilidad y variables de salud psicosocial, así como explorar el papel de la empleabilidad y la inseguridad laboral en la salud psicosocial. Para esto, se utilizó metodología cuasiexperimental, donde se adminis- traron una serie de cuestionarios que evaluaban empleabilidad e inseguridad laboral a 97 trabajadores (35 desempleados y 62 en activo), junto a otras variables psicosociales y de salud. Los resultados muestran que la empleabilidad es mayor en desempleados y que, altas puntuaciones en este constructo se asocian a un menor malestar afectivo. Por otro lado, en la población activa, tener altos niveles de inseguridad laboral también se asocian con malestar psicológico y baja satisfacción y autoeficacia. Estos resultados se discuten considerando que la empleabilidad podría estar actuando como un amortiguador de las consecuencias negativas derivadas del desempleo y la inseguridad laboral.

Correspondencia: Miguel Angel Serrano Rosa. Departamento de Psicobiología. Universitat de València.

Email: m.angel.serrano@uv.es

ORCID: Miguel Angel Serrano Rosa (http://orcid.org/0000-0002-6574-4532), Sandra Baena (http://orcid.org/0000-00028563-3186) y Francisco Molins-Correa (http://orcid.org/0000-0002-9763-3132)

${ }^{1}$ Universitat de València, España.

Recibido: 22 de enero de 2018 .

Aceptado: 23 de mayo de 2018 . 
Palabras clave: Empleabilidad; Inseguridad laboral; Salud; Desempleo; Trabajadores.

\begin{abstract}
Unemployment and job insecurity have negative effects on health, although employability may be able to mediate these effects. The objective of this article is to analyze the possible differences between workers and unemployed in employability and psychosocial health variables, as well as to explore the role of employability and job insecurity in the active population in psychosocial health. For this, quasiexperimental methodology was used, where a questionnaire evaluating employability, job insecurity and psychosocial health variables was administered to 97 workers (35 unemployed and 62 in active). Results showed that employability is higher in unemployed and that high scores in this construct are associated with negative emotions. On the other hand, in the active population, having high levels of job insecurity were associated with psychological distress and low satisfaction and self-efficacy. These results are discussed considering that employability could be buffer the negative health consequences derived from unemployment and job insecurity.
\end{abstract}

Keywords: Employability; Job Insecurity; Health; Unemployment; Workers.

\section{Introducción}

El avance de las nuevas tecnologías y la globalización de los mercados ha supuesto una transición desde las formas tradicionales de capitalismo hasta el denominado "Capitalismo flexible" que impera actualmente (Sennett, 2006). En el Capitalismo tradicional, el trabajo solía tener una "estructuración lineal y acumulativa del tiempo" -más conocida como trayectoria laboral-, y el lugar de trabajo era "estable y único", sin grandes modificaciones durante la vida del trabajador. Esto confería seguridad laboral y favorecía el desarrollo de la propia identidad del individuo (Valenzuela, 2014). En el actual Capitalismo flexible, sin embargo, aparecen nuevas prácticas laborales, como la flexibilización, la externalización, el trabajo en equipos temporales, la subcontratación, etc. (Valenzuela, 2014), caracterizadas por su flexibilidad, falta de estabilidad y el lema de "nada es a largo plazo" (Sisto, 2009; Valenzuela, 2014). Éstas establecen unas relaciones laborales opresivas y desorientadoras (Rivas, 2006; Sennett, 2006) y suponen una precarización del trabajo (Rivas, 2006; Standing, 2011; Valenzuela, 2014), jugando en contra del desarrollo de la identidad y produciendo inseguridad laboral.

Este cambio también pone toda la responsabilidad de la contratación sobre el trabajador, exigiéndole ahora absoluta disponibilidad, -el "lastre cero" (Hochschild, 1997) en su forma más extrema-, "un comportamiento ágil" (Sennett, 2006) y que esté siempre en las mejores condiciones profesionales, intelectuales, físicas, etc. Es decir, que sea un producto atractivo y susceptible de ser contratado (Bauman, 2007).

Además, la crisis financiera mundial que dio comienzo a finales de 2007 -aunque sus efectos sobre el mercado laboral empezaran a finales del 2008-, trajo consigo una importante disminución del trabajo -de 2008 a 2012, en España, se destruyeron 7,2 millones de puestos, creándose tan sólo 2,4 millones-, incrementando aún más el deterioro de las condiciones del empleo existente (Rocha y Aragón, 2012) y dejando a muchos trabajadores en paro.

De este complicado contexto se deriva, por un lado, (1) que la situación laboral actual dificulta la reincorporación de las personas en paro, quedando más expuestas al factor de riesgo psicológico que supone el desempleo. Éste se asocia al aumento o exacerbación de las enfermedades mentales tales como los cuadros ansioso-depresivos, los trastornos somatomorfos y/o del sueño; las conductas de riesgo como el consumo de alcohol, tabaco y otras sustancias; así como los conflictos familiares y los casos de suicidio (e.g., Allebeck, 2013; Bernal, Gasparrini, Artundo y McKee; 2013; Granado, 2014; Salvador-Carrulla y Roca, 2013), aunque Gili, Campayo y Roca (2014), señalen que el aumento de los últimos no es significativo. Por otra parte, (2) la inseguridad laboral que producen las nuevas formas de trabajo, entendida como el miedo a perder el empleo, puede ocasionar en la población activa una pérdida de la satisfacción laboral (Sverke, Hellgren y 
Näswall, 2002), un sentimiento de angustia (Valenzuela, 2014) y, como afirma Granado (2014), un estrés comparable a la pérdida del trabajo en sí, relacionado con una pobre salud psicológica.

Ante esta situación, se hace evidente la necesidad de buscar estrategias que reduzcan los efectos nocivos derivados del desempleo y la inseguridad laboral. Y es aquí donde cabe subrayar el concepto de empleabilidad. La empleabilidad es un constructo cuya concepción no ha sido igual para todos los teóricos. En su origen, muchos autores la entendieron como una habilidad personal para incorporarse al mundo laboral, permanecer en un empleo u obtener uno nuevo (e.g., Hillage y Pollard, 1998; Rousseuau, 1997). De Grip, van Loo, y Sanders (2004) hablan de la voluntad y capacidad que tiene un trabajador para tratar de ser atractivo para las organizaciones, teniendo una actitud de anticipación y reacción a los cambios del entorno laboral. Y, en la misma línea, Fugate et al. (2004), hablan de la empleabilidad como un término centrado en varios factores personales que constituyen, concretamente, dos meta-habilidades: (1) la proactividad y (2) la identidad profesional, ambas necesarias para adaptarse y gestionar la carrera profesional y que, en dicha adaptación, se integre el capital humano y social (Nazar y VanDer Heijden, 2012); es decir, que el individuo se identifique y comprometa con el trabajo.

Hoy sabemos que la empleabilidad puede ser dividida en dos dimensiones (Bargsted, 2008): (1) la empleabilidad objetiva o conjunto de aspectos objetivos, tales como la jerarquía, las condiciones del contrato, la estabilidad del mercado laboral, entre otras variables observables (Bargsted, 2017), es decir, las condiciones reales del contexto; y (2) la empleabilidad subjetiva, como proponen Vanhercke et al (2014), entendida como la medida subjetiva de la creencia que tienen los individuos sobre la facilidad para encontrar un nuevo trabajo que, a su vez, supone un factor crucial para la consecución del mismo, así como del éxito laboral (De Cuyper et al., 2011a, b, 2014; Kirves et al., 2014).

En un mercado laboral deteriorado, donde la empleabilidad objetiva se ve disminuida (Bargsted, 2017), y donde el foco de la contratación se pone en los propios individuos, parece interesante, por tanto, centrarse en tra- bajar con los trabajadores para lograr un incremento de su empleabilidad percibida (Bargsted, 2017); es decir, focalizarse en desarrollar la empleabilidad subjetiva que confiera a los trabajadores de un mayor potencial para la consecución o mantenimiento del empleo.

Además de la relación positiva que parece encontrarse entre la empleabilidad y las posibilidades de obtener empleo o mantenerlo, existe otra línea de investigación que habla de ésta como variable amortiguadora de las consecuencias nocivas que producen el desempleo y la inseguridad laboral, debido a su relación positiva con otros factores como la autoeficacia y el bienestar afectivo (McArdle, Waters, Briscoe y Hall, 2007) o la satisfacción laboral, el bienestar y el estado de salud general (e.g., Bernston y Marklund, 2007; De Cuyper, Bernhard-Oettel, Bernston, De Witte y Alarco, 2008; Gamboa, Gracia, Ripoll y Peiró, 2007), aportando un valor añadido a favor de la promoción de la empleabilidad.

Por tanto, ante el actual aumento del desempleo y ante la inseguridad laboral que producen las nuevas formas de trabajo, parece interesante desarrollar la empleabilidad tanto en desempleados como en población activa. Así, los parados, se beneficiarían de un mayor potencial en la búsqueda de empleo y reducirían el riesgo a padecer enfermedades mentales (McArdle et al., 2007; Vanhercke et al., 2014); y, por su parte, los trabajadores, reducirían los efectos nocivos que produce la inseguridad laboral, prevendrían la pérdida de su trabajo y, en caso de pérdida, estarían preparados para afrontar proactivamente la situación de desempleo, agilizando su reincorporación (Bernston y Marklund, 2007; De Grip, van Loo y Sanders, 2004). No obstante, es necesario indicar que, aunque la empleabilidad pueda incrementar en gran medida las probabilidades de encontrar un empleo, todo dependerá de las condiciones económicas y oportunidades laborales que existan.

Por tanto, nuestro estudio tiene como objetivo evidenciar el papel de la empleabilidad como variable que puede influir en la inseguridad laboral y el tener/o no tener empleo y en sus efectos a nivel psicológico. Esperamos contrastar las siguientes hipótesis:

H1. Las personas en desempleo puntuarán más en empleabilidad que la población activa. 
H2. Las personas en desempleo presentarán mayor malestar psicológico e insatisfacción, así como menor autoeficacia y optimismo, en comparación con los empleados. H3. Por otro lado, las personas que tengan altas puntuaciones en empleabilidad presentarán menor malestar afectivo y mayores puntuaciones en satisfacción, salud general, autoeficacia y optimismo -variables positivas en adelante-(Bernston y Marklund, 2007; De Cuyper, BernhardOettel, Bernston, De Witte y Alarco, 2008; Gamboa, Gracia, Ripoll y Peiró, 2007; McArdle, Waters, Briscoe y Hall, 2007), así como mayor expectativa de encontrar trabajo en los próximos meses, que los que presentan bajos niveles en empleabilidad.

H4. Por último, entre la población activa, hipotetizamos que los trabajadores que perciban su situación laboral como más insegura, puntuarán más en malestar afectivo y menos en las variables positivas que quienes perciban menor inseguridad laboral (Sverkeet al., 2002).

H5. Además, hipotetizamos que el grado de empleabilidad influirá en los niveles de inseguridad laboral en la población activa.

\section{Método}

\section{Participantes}

La muestra se compone de 97 participantes. El rango de edad va desde los 18 hasta los 62 años. Un $48.5 \%$ son hombres y el $51.5 \%$ son mujeres. En relación a las cargas familiares, encontramos que un $54.6 \%$ tienen familiares a su cargo, mientras que el $45.4 \%$ no tienen cargas. Las cargas económicas dividen a los participantes entre los que tienen actualmente una hipoteca, el $26.8 \%$ del total; los que tienen un préstamo, el 19.6\%; los que comparten ambas cargas, $15.5 \%$; y los que no tienen ninguna carga económica de este tipo actualmente, que son el $38.1 \%$.

En cuanto a la situación laboral se refiere, la muestra se divide en un $63.9 \%$ de personas que en activo - 62 participantes-, y un $36.1 \%$ desempleados -35 participantes-

Del subconjunto de personas en activo, todos trabajaban en una misma zona de la comunidad valenciana atenazada por la crisis laboral y pertenecían a una industria del sector agrario. La duración media de permanencia en la empresa es de aproximadamente cinco años y tres meses, y el tiempo que estuvieron en paro antes de encontrar su actual trabajo se establece en siete meses. En cuanto al tipo de contrato, la muestra se divide en un $27.4 \%$ con un contrato de duración determinada, un $48.4 \%$ con duración indeterminada y un $24.2 \%$ con otro tipo de contrato diferente. Por otro lado, en personas desempleadas, el tiempo medio de paro se establece en dos años y dos meses y la esperanza de encontrar un trabajo en los próximos meses se divide en: $14.3 \%$ que no cree que pueda encontrarlo, un $25.7 \%$ que considera que existen pocas posibilidades, un $25.7 \%$ que afirma no saber si lo encontrará o no, un $20 \%$ que considera que existen muchas posibilidades, y un $14.3 \%$ que están seguros de encontrarlo pronto. Se comprobó que no había diferencias significativas en edad, ni en cargas familiares ni económicas.

Este estudio se realizó en 2015 y basa en un muestreo a propósito, no probabilístico, dado que los participantes se buscaron mediante contactos de personas conocidas a través de las empresas en una zona industrial/agraria de la comunidad valenciana. Este tipo de muestreo no asegura una representatividad de la población como la que pueda tener un muestreo probabilístico, pero, teniendo en cuenta el carácter exploratorio de este estudio consideramos que puede ser útil para responder a nuestros objetivos.

La participación ha sido completamente voluntaria y protegida, puesto que los datos se han tratado de forma anónima y con la confidencialidad y rigurosidad pertinentes según las normas éticas de protección de datos de la Ley Orgánica 15/1999 del 13 de diciembre, sobre la regulación del tratamiento automatizado de datos sobre carácter personal.

\section{Material}

Se utilizaron dos modelos de cuestionarios adaptando las variables que miden en función de cada subconjunto de la muestra -trabajadores y desempleados-. Éstas son de dos tipos, (1) variables socio-demográficas: edad, género, presencia de cargas familiares y/o económicas, duración de desempleo o del último paro laboral, duración en la última empresa o en la empresa actual, tipo de contrato y esperanza de encontrar empleo próximamente (ésta úl- 
tima, únicamente para el grupo de desempleados); y (2) las siguientes variables rasgo/situacionales: Autoeficacia general, medida mediante la adaptación española de la Escala de Autoeficacia General de Baessler y Schwarzer (1996). Consta de 10 ítems en escala Likert de cuatro puntos, y evalúa el sentimiento estable de competencia personal para manejar de forma eficaz gran variedad de situaciones estresantes. La fiabilidad obtenida en nuestra muestra es de .908 (alfa de Cronbach); Optimismo disposicional o predisposición a las expectativas de resultados positivos o negativos. Se ha utilizado el Test Revisado de Orientación Vital de Scheier, Carver y Bridges (1994), en su versión española -LOT-R- validada por Otero, Luengo, Romero, Gómez y Castro (1998). Se compone de 10 ítems, seis valoran optimismo -tres en positivo y tres en negativo-, y los otros cuatro son distractores. La fiabilidad obtenida en nuestra muestra es de .770 (alfa de Cronbach); Satisfacción laboral, registrada con la Escala de Brayfield y Rothe (1951), compuesta por cuatro ítems y cinco alternativas de respuesta tipo Likert. La fiabilidad obtenida en nuestra muestra es de .875 (alfa de Cronbach); Malestar afectivo -asociado al trabajo-, medido mediante la escala de Warr (1990). Formada por 12 ítems tipo Likert que constituyen dos factores, seis para medir estados de ánimo depresivo y seis para medir ansiedad. La fiabilidad obtenida en nuestra muestra es de .913 (alfa de Cronbach); y Salud general percibida mediante el Cuestionario de Salud General GHQ-12- de Goldberg (1979), adaptado al español por Lobo, Pérez-Echeverría y Artal (1986). Se trata de una versión reducida de 12 ítems que valora la percepción del estado de salud general de la persona, para población adulta. La fiabilidad obtenida en nuestra muestra es de .044 (alfa de Cronbach).

Además, como variables principales de este estudio, se midió empleabilidad y la inseguridad laboral. La Escala de Empleabilidad desarrollada por De Witte (1992) consta de 5 ítems en formato Likert y se pasó a los dos grupos. Se refieren a las posibilidades que el individuo cree tener sobre si encontraría otro trabajo en caso de buscarlo (tanto si se tiene trabajo como si no). La fiabilidad obtenida en nuestra muestra es de .807 (alfa de Cronbach). Por último, la escala de Inseguridad laboral, desarrollada por De Witte (2000), y utilizando la traducción de Peiró (2007) consta de cuatro ítems con cinco alternativas en escala tipo
Likert. Este instrumento mide la probabilidad percibida por el individuo de perder el propio trabajo y sólo fue administrado al grupo de empleados activos. La fiabilidad obtenida en nuestra muestra es de .845 (alfa de Cronbach).

\section{Procedimiento}

La metodología de este estudio exploratorio fue cuasiexperimental. Así, tras la adaptación de los cuestionarios en función de los dos subgrupos en que se divide la muestra-trabajadores y desempleados-, se distribuyeron entre los participantes del estudio en formato de papel impreso. Se les pedía a estos que cumplimentaran los formularios tal y como se indicaba en las instrucciones, al inicio de cada uno de ellos y se entregaran en un periodo de una semana.

\section{Análisis estadísticos}

En primer lugar, se comprobó la normalidad y homocedasticidad de las variables, con las pruebas de Kolmogorov y Levene, respectivamente. Dado que las variables evaluadas, a excepción del optimismo, no se distribuían normalmente se decidió utilizar estadística no paramétrica para responder a las hipótesis.

Para contrastar las hipótesis indicadas, en primer lugar, se comprobó si había diferencias entre los trabajadores y los desempleados en todas las variables anteriores, mediante la $U$ de Mann-Whitney para muestras independientes. Posteriormente, se utilizó, basándonos en el rango de percentiles, la mediana con el fin de dividir la muestra en alta y baja empleabilidad, y alta y baja inseguridad laboral en el caso de los trabajadores en activo. Para contrastar las hipótesis especificadas se utilizaron pruebas $U$ de MannWhitney. Todos los análisis han sido realizados con el paquete estadístico SPSS 20, especificando un nivel de significación de 0.05 . 
Tabla 1

Medias y desviaciones típicas y estadísticos de contraste ( $U$ de Mann-Whitney) para personas en activo y en paro en empleabilidad, satisfacción, malestar afectivo (ansiedad y depresión), salud general, autoeficacia y optimismo

\begin{tabular}{lcccc}
\hline Situación laboral & En activo & Desempleo & z & Sig. (bilateral) \\
\hline Empleabilidad & $28.43(5.58)$ & $31.18(4.56)$ & -7.00 & .001 \\
Satisfacción & $14.79(3.72)$ & $13.48(4.32)$ & -1.55 & .121 \\
Depresión & $10.74(3.63)$ & $12.25(4.74)$ & -1.37 & .170 \\
Ansiedad & $13.87(4.39)$ & $14.45(6.18)$ & -0.42 & .673 \\
Salud general & $32.00(3.97)$ & $34.51(2.88)$ & -3.16 & .002 \\
Autoeficacia & $30.33(5.34)$ & $31.74(7.32)$ & -1.43 & .152 \\
Optimismo & $14.62(4.54)$ & $16.25(5.71)$ & -1.79 & .073 \\
\hline
\end{tabular}

\section{Resultados}

\section{Situación laboral, empleabilidad y salud psicosocial}

La Tabla 1 muestra que las personas en situación de desempleo presentan una puntuación estadísticamente más elevada en empleabilidad que la población activa. También, se puede observar que las personas desempleadas presentan una puntuación en salud general percibida significativamente más elevada que las personas en activo. Por último, la variable optimismo presenta una tendencia a la significación siendo mayor la puntuación en desempleados. El resto de variables -satisfacción, malestar afectivo, ansiedad y autoeficacia - no presentan diferencias significativas $(p>0.1)$.

\section{Empleabiliadda y variables psicológicas relacionadas}

En el caso de la empleabilidad, podemos observar (Tabla 2) que las personas que puntúan alto en este constructo, presentan niveles de satisfacción, significativamente más elevados que las personas con baja empleabilidad. Además, los niveles de malestar afectivo (parámetros de depresión y ansiedad) son estadísticamente más altos en las personas con una empleabilidad baja. Respecto a la salud general percibida, no se aprecian diferencias significativas $(p>0.1)$.

\section{Inseguridad laboral y variables psicológicas relacionadas}

En cuanto a la inseguridad laboral (Tabla 3) se puede comprobar cómo los trabajadores que presentan puntua-

Tabla 2

Medias y desviaciones típicas y estadísticos de contraste ( $U$ de Mann-Whitney) para personas con empleabilidad alta y baja en los factores de satisfacción, malestar afectivo (depresión y ansiedad), salud general, autoeficacia y optimismo

\begin{tabular}{lcccc}
\hline Empleabilidad & Alta empleabilidad & Baja empleabilidad & z & Sig. (bilateral) \\
\hline Satisfacción & $15.40(3.80)$ & $13.70(3.97)$ & -2.59 & .009 \\
Depresión & $9.60(3.33)$ & $12.24(4.22)$ & -2.50 & .012 \\
Ansiedad & $11.68(4.81)$ & $15.43(4.75)$ & -2.04 & .041 \\
Salud general & $33.51(4.00)$ & $32.56(3.67)$ & -1.68 & .092 \\
Autoeficacia & $33.02(5.80)$ & $29.61(6.01)$ & -0.99 & .318 \\
Optimismo & $16.29(4.83)$ & $14.29(4.93)$ & -0.33 & .741 \\
\hline
\end{tabular}


Tabla 3

Medias y desviaciones típicas y estadísticos de contraste ( $U$ de Mann-Whitney) para personas empleadas con puntuaciones altas y bajas en inseguridad laboral, en los factores de satisfacción, malestar afectivo, salud general, autoeficacia y optimismo

\begin{tabular}{lcccc}
\hline Inseguridad laboral & $\begin{array}{c}\text { Alta Inseguridad } \\
\text { laboral }\end{array}$ & $\begin{array}{c}\text { Baja Inseguridad } \\
\text { laboral }\end{array}$ & $\mathbf{z}$ & Sig. (bilateral) \\
\hline Satisfacción & $12.50(4.19)$ & $16.23(2.54)$ & -3.07 & .002 \\
Depresión & $12.25(3.65)$ & $9.78(3.30)$ & -2.19 & .028 \\
Ansiedad & $16.37(4.30)$ & $12.28(3.67)$ & -3.03 & .002 \\
Salud general & $33.16(3.34)$ & $31.26(4.20)$ & -2.20 & .028 \\
Autoeficacia & $27.12(5.35)$ & $32.36(4.28)$ & -2.82 & .005 \\
Optimismo & $13.83(5.24)$ & $15.13(4.02)$ & -1.64 & .100 \\
\hline
\end{tabular}

ciones elevadas en inseguridad, tienen significativamente puntuaciones más bajas en satisfacción laboral y autoeficacia. Además, tienen mayores puntuaciones en depresión, ansiedad y salud general. Los niveles de optimismo no son diferentes significativamente entre grupos $(p>0.1)$.

Para terminar, los análisis entre inseguridad laboral y empleabilidad evidencian que, del subconjunto de personas en activo, aquellas con alta empleabilidad, tienen puntuaciones en inseguridad laboral significativamente más bajas que las personas con baja empleabilidad $(U=-154$, $p<.000)$.

\section{Discusión}

Antes de iniciar la discusión de los resultados es importante indicar que este estudio se realizó en el año 2015, en una zona industrial/agraria que se vio afectada en gran medida por la crisis económica iniciada en 2009. Así todos los resultados obtenidos, y que a continuación se discutirán, deben observarse bajo el prisma de una población que ha sufrido de lleno una crisis económica.

Así, en primer lugar, sustentando la hipótesis inicial, se observa que las personas en situación de desempleo tienen una mayor empleabilidad que los que se encuentran actualmente trabajando. Este resultado podría interpretarse teniendo en cuenta la necesidad de los desempleados de buscar estrategias para incrementar las posibilidades de encontrar un empleo; es decir, los desempleados deben de- dicar más esfuerzo y tiempo en fomentar sus capacidades laborales con el fin de poder cambiar de estatus laboral y poderse incorporar al mercado laboral. Por otro lado, hipotetizamos que estas diferencias podrían estar maximizándose si el trabajador que está en activo no dedica tiempo (o no tiene tiempo) a fomentar su empleabilidad. Esta última idea debería comprobarse y de ser así, las autoridades deberían plantearse métodos de fomentar la empleabilidad en la población activa. De hecho, esta idea iría en línea con las dificultades que, según Rentería, Perez y Malvezzi (2008), encuentra esta población para acceder a los programas de empleabilidad y evidenciaría la necesidad de replantearse las prioridades y buscar nuevos enfoques que favorezcan también a la población activa, por ejemplo, desarrollando nuevos programas de empleabilidad que los tengan en mayor consideración. Por otro lado, contrariamente a la hipótesis de partida, los desempleados presentan mayor percepción de salud general y optimismo. Este resultado podría estar sustentando la idea que trabajar tiene perjuicios para la salud psicosocial. De hecho, la Ley de Prevención de Riesgos Laborales trata de combatir los efectos negativos del trabajo sobre la salud. Además, esto apoya los planteamientos de Granado (2014) acerca de la similitud entre los efectos nocivos de las condiciones laborales y los del desempleo.

Contribuyendo ahora a ampliar las evidencias aportadas anteriormente en este sentido (McArdle et al., 2007) y cumpliendo nuestra hipótesis, hallamos de nuevo cómo tener alta empleabilidad se relaciona con las variables de salud psicosocial. Concretamente, vemos cómo aquellos que destacan en este constructo, tienen menores puntuaciones en ansiedad y depresión, y mayores puntaciones en satis- 
facción, en comparación con aquellos con menor empleabilidad. Tal vez por esto, por el mejor estado de ánimo y la mayor empleabilidad, sea por lo que las personas desempleadas con mayor puntuación en la misma presenten, además, una mayor esperanza de encontrar en empleo, clave según De Cuyper et al. (2011a, b, 2014) y Kirves et al. (2014) para encontrarlo realmente.

Por último, y centrándonos en la población activa, podemos comprobar que, efectivamente, como ya anticiparon otros autores (Granado, 2014; Sverke et al., 2002; Valenzuela, 2014), así como nosotros predijimos en nuestras hipótesis, la inseguridad laboral se relaciona con efectos nocivos como el propio desempleo. Así, quien percibe su empleo como más inseguro, muestra también un mayor malestar afectivo y, en consecuencia, una menor satisfacción laboral. Quizás sea por su sentimiento de indefensión ante la posible pérdida del trabajo por lo que, además, se percibe como menos autoeficaz. Sin embargo, aun pareciendo incongruente, no se observan diferencias significativas en cuanto a su optimismo y tampoco en autoeficacia. Por último, podemos añadir todavía más pruebas sobre la relación que tiene la empleabilidad con la inseguridad laboral. Así, quienes muestran mayor empleabilidad, tienen a su vez menores puntuaciones en inseguridad laboral, con su consecuente reducción, por tanto, en todos los efectos negativos que asociábamos con ésta (Sverke et al., 2002; Valenzuela, 2014).

Las implicaciones que podrían tener nuestros resultados serían, en primer lugar, la necesidad de comprobar la hipótesis de si la población activa tiene carencias en empleabilidad y por tanto hubiera que fomentarla, sobre todo, por reducir la inseguridad laboral, tal y como se observa también en los resultados. Desde nuestro punto de vista, el mercado laboral sufre constantes transformaciones que puede dejar obsoletas las capacidades/habilidades de cualquier trabajador, por lo que sería necesario tener trabajadores en continua formación. La segunda implicación que tienen nuestros resultados va más allá de la mera obtención de un empleo o su mantenimiento, ya que hemos observado que tener alta o baja empleabilidad e inseguridad podría estar influyendo en los niveles de malestar psicológico, satisfacción y optimismo. Es decir, fomentar la empleabilidad y reducir la inseguridad reduciría la probabilidad de alteraciones del estado de ánimo y por tanto afectarían positivamente la salud. Por tanto, dentro de un plan- teamiento preventivo el fomento de la empleabilidad permitiría tener trabajadores con más capacidades para encontrar o mantener un empleo además de tener una mejor salud emocional. En este sentido, las políticas de inserción laboral deberían tener en cuenta estos resultados para justificar con más fuerza la necesidad formativa para el aumento de la empleabilidad, no sólo por razones laborales sino de salud.

Cabe señalar, no obstante, que este estudio tiene ciertas limitaciones. Por un lado, sería conveniente utilizar una muestra mayor y que abarcase otros sectores laborales y zonas geográficas, aumentando su validez ecológica. También sería interesante poder realizar estudios prospectivos para, de este modo, distinguir los factores de riesgo en relación con la inseguridad laboral y otras variables, de los factores internos de personalidad, lo que permitiría hacer inferencias de causalidad entre estos factores. Además, sería conveniente validar escalas de empleabilidad e inseguridad laboral con el fin de tener mejores medidas e estos constructos tan importantes. Finalmente, otra limitación sería la estrategia de análisis, a la hora de dividir los grupos, dado que hay literatura en contra de esta práctica, pero, en cambio, hay publicaciones que utilizan la misma. En nuestro caso, hemos optado por utilizarla dado que nos permitía separa la población en dos grupos para comprobar nuestras hipótesis. No obstante, en futuros estudios, con más muestra, se podrían probar otras estrategias estadísticas más potentes.

\section{Conclusiones}

Los datos de nuestro estudio destacan las diferencias en empleabilidad y salud entre trabajadores y empleados, así como resaltan la importancia de la empleabilidad como factor clave, tanto en trabajadores como en desempleados, ante el contexto de Capitalismo flexible que se respira en la actualidad, no sólo por su papel facilitador en la obtención o mantenimiento del empleo sino, además, por su efecto beneficioso sobre la salud, previamente señalado por numerosos autores (Bernston y Marklund, 2007; De Cuyper, Bernhard-Oettel, Bernston, De Witte y Alarco, 2008; Gamboa, Gracia, Ripoll y Peiró, 2007; McArdle et al., 2007;). Por otro lado, la percepción de inseguridad laboral también se presenta como un aspecto clave a la hora 
de interpretar el estado emocional de los trabajadores que podría modularse fomentando la empleabilidad. En conclusión, desde nuestro punto de vista y a la luz de los resultados, consideramos que se debería hacer más hincapié en el fomento de la empleabilidad como factor clave para la obtención de empleo y tener mejor salud emocional.

\section{Referencias}

Allebeck, P. (2013). Health Effects of the Crisis: Challenges for Science and Policy. European Journal of Public Health, 23(5), 721. Recuperado de https://academ-

ic.oup.com/eurpub/article/23/5/721/450531

Baessler, J. y Schwarzer, R. (1996). Evaluación de la autoeficacia: Adaptación española de la escala de Autoeficacia General [Evaluation of Self-Efficacy: Spanish Adaptation of the General Self-efficacy scale]. Ansiedad y Estrés, 2(1), 1-8.

Bargsted, M. (2008). Éxito integral en la carrera laboral: congruencia entre valores y metas [Integral Success in the Work Career: Congruence between Values and Goals] (Tesis doctoral no publicada). Universidad Autónoma de Madrid. Madrid, España.

Bargsted, M. (2017). Impact of Personal Competencies and Market Value of Type of Occupation over Objective Employability and Perceived Career Opportunities of Young Professionals. Journal of Work and Organizational Psychology, 33(2), 115123. https://doi.org/10.1016/j.rpto.2017.02.003

Bauman, Z. (2007). Vida de consumo [Consumption Life]. Buenos Aires, Argentina: Fondo de cultura económica.

Bernal, J., Gasparrini, A., Artundo, C. y McKee, M. (2013). The Effect of the Late 2000s Financial Crisis on Suicides in Spain: An Interrupted Timeseries Analysis. European Journal of Public Health, 23(5), 732. Recuperado de https://doi.org/10.1093/eurpub/ckt083

Berntson, E. y Marklund, S. (2007). The Relationship between Perceived Employability and Subsequent Health. Work \& Stress, 21(3), 279-292. https://doi.org/10.1080/02678370701659215 
Brayfield, A. H. y Rothe, H. F. (1951). An Index of Job Satisfaction. Journal of Applied Psychology, 35(5), 307.

De Cuyper, N., Bernhard-Oettel, C., Berntson, E., Witte, H. D. y Alarco, B. (2008). Employability and Employees' Well-Being: Mediation by Job Insecurity. Applied Psychology, 57(3), 488-509. https://doi.org/10.1111/j.1464-0597.2008.00332.x

De Cuyper, N., Van der Heijden, B. I. J. M. y De Witte, H. (2011a). Associations between Employability and Employee and Organizational Outcomes: A Matter of Psychological Contracts? International Journal of Human Resource Management, 22(7), 1486-1503.

https://doi.org/10.1080/09585192.2011.561962

De Cuyper, N., Mauno, S., Kinnunen, U. y Mäkikangas, A. (2011b). The Role of Job Resources in the Relation between Perceived Employability and Turnover Intention: A Prospective Two-Sample Study. Journal of Vocational Behavior, 78(2), 253263. https://doi.org/10.1016/j.jvb.2010.09.008

De Cuyper, N., Sulea, C., Philippaers, K., Fischmann, G., Iliescu, D. y De Witte, H. (2014). Perceived Employability and Performance: Moderation by Felt Job Insecurity. Personnel Review, 43(2), 536552. https://doi.org/10.1108/PR-03-2013-0050

De Grip, A., van Loo, J. y Sanders, J. (2004). The Industry Employability Index: Taking Account of Supply and Demand Characteristics. International Labour Review, 143(3), 211-233.

De Witte, H. (1992). Tussen optimisten en teruggetrokkenen. Een empirisch onderzoek naar het psychosociaal profiel van langdurig werklozen en deelnemers aan de Weer-Werkactie in Vlaanderen [Between Optimists and Retreats. An Empirical Study of the Psychosocial Profile of the Long-Term Unemployed and Participants in the Weather Action in Flanders]. Lovaina: HIVA.
De Witte, H. (2000). Arbeidsethos en jobonzekerheid: Meting en gevolgen voor welzijn, evredenheid en inzet op het werk [Work Ethic and Job Insecurity: Measurement and Consequences for Wellbeing, Evasiveness and Commitment to Work]. En R. Bouwen, K. De Witte, H. De Witte y T. Taillieu (Eds.), Van groep naar gemeenschap (pp. 325350). Leuven, Bruselas: Garant.

Fugate, M., Kinicki, A. y Asforth, B. (2004). Employability: A Psychosocial Construct, its Dimensions, and Applications. Journal of Vocational Behavior, 65, 14-38. https://doi.org/10.1016/j.jvb.2003.10.005

Gamboa, J., Gracia, F., Ripoll, P. y Peiró, J. M. (2007). La empleabilidad y la iniciativa personal como antecedentes de la satisfacción laboral [Employability and Personal Initiative as a Background to Job Satisfaction]. Valencia, España: Instituto Valenciano de Investigaciones Económicas. Recuperado de http://www.ivie.es/downloads/docs/wpasec/wpase c-2007-01.pdf.

Gili, M., Campayo, J. G. y Roca, M. (2014). Crisis económica y salud mental. Informe SESPAS 2014 [Economic Crisis and Mental Health. SESPAS report 2014]. Gaceta Sanitaria, 28, 104-108. https://doi.org/10.1016/j.gaceta.2014.02.005

Goldberg D. P. y Hillier V. F. (1979). A Scaled Version of the General Health Questionnaire. Psychological Medicine, 9, 139-145.

Granado, A. (2014). Crisis económica, políticas, desempleo y salud (mental) [Economic Crisis, Politics, Unemployment and (Mental) Health]. Revista de la Asociación Española de Neuropsiquiatría, 34(122), 385-404. http://dx.doi.org/10.4321/S021157352014000200010

Hillage, J., y Pollard, E. (1998). Employability: Developing a Framework for Policy Analysis. 
London: Department for Education and Employment.

Hochschild, A. R. (1997). The Time Bind: When home Becomes Work and Work Becomes Home. New York: CA Henry Holt.

Jacobson, D. (1991). Toward Theoretical Distinction between the Stress Components of the Job Insecurity and Job Loss Experiences. Research in the Sociology of Organizations, 9, 1-19.

Kirves, K., Kinnunen, U., De Cuyper, N. y Mäkikangas, A. (2014). Trajectories of Perceived Employability and their Associations with Well-Being at Work: A Three-Wave Study. Journal of Personnel Psychology, 13(1), 46-57. https://doi.org/10.1027/1866-5888/a000103

Lobo A., Pérez-Echeverría M. J. y Artal J. (1986). Validity of the Scaled Version of the General Health Questionnaire (GHQ-28) in a Spanish Population. Psychological Medicine, 16, 135-140.

McArdle, S., Waters, L., Briscoe, J. P. y Hall, D. T. T. (2007). Employability during Unemployment: Adaptability, Career Identity and Human and Social Capital. Journal of Vocational Behavior, 71(2), 247-264. https://doi.org/10.1016/j.jvb.2007.06.003

Nazar, G., y Van der Heijden, B. M. (2012). Career Identity and its Impactupon Self-Perceived Employability among Chilean male middleAgedmanagers. Human Resource Development International, 15(2), 141-156. https://doi.org/10.1080/13678868.2012.664692

Otero, J. M., Luengo, A. y Romero, E. G. J. A. y Castro, C. (1998). Psicología de personalidad. Manual de o [Personality Psychology. Practices Manual]. Barcelona, España: Ariel Prácticum.

Rentería-Pérez, E. y Malvezzi, S. (2008). Empleabilidad, cambios y exigencias psicosociales en el trabajo [Employability, Changes and
Psychosociological Demands on Work]. Universitas Psychologica, 7(2), 319-334. Recuperado de http://revistas.javeriana.edu.co/index.php/revPsyc ho/article/view/319/248

Rivas, A. M. (2006). El empleo o la vida: perder el empleo para conservar la vida o renunciar a la vida para conservar el empleo ¿de qué conciliación hablamos? [Employment or life: Losing one's Job to Keep one's life or Giving up one's Life to Keep one's Job. What Conciliation are we Talking about?] Revista de Antropología Iberoamericana, 1(3), 261-368. Recuperado de http://www.redalyc.org/pdf/623/62310302.pdf

Rocha, F. y Aragon, J. (2012). La crisis económica y sus efectos sobre el empleo en España [The Economic Crisis and its Effects on Employment in Spain]. Gaceta Sindical, 19, 67-90. Recuperado de http://docpublicos.ccoo.es/cendoc/035344CrisisEc onomicaEfectos.pdf

Rousseau, D. M. (1997). Organizational Behaviour in the new Organizational Era. Annual Review of Psychology, 48(1), 515-546. https://doi.org/10.1146/annurev.psych.48.1.515

Salvador-Carulla L., y Roca M., (2013). Mental Health Impact of the Economic Crisis in Spain. Intern Psychia 10(1), 8-10.

Scheier, M., Carver, C. y Bridges, M. (1994). Distinguishing Optimism from Neuroticism (and Trait Anxiety, Self-Mastery, and Self-Estem): A Reevaluation of the Life Orientation Test. Journal of Personality and Social Psychology, 67, 10631078. 3514.67.6.1063

Sennett, R. (2006). La corrosión del carácter: las consecuencias personales del trabajo en el nuevo capitalismo [Character Corrosion: The Personal Consequences of Work in the New Capitalism]. Barcelona, España: Anagrama. 
Sisto, V. (2009). Cambios en el trabajo, identidad e inclusión social en Chile: desafíos para la investigación [Changes in Work, Identity and Social Inclusion in Chile: Challenges for Research]. Universum, 24(2), 192-216. http://dx.doi.org/10.4067/S071823762009000200011

Sverke, M., Hellgren, J. y Näswall, K. (2002). No Security: A Meta-Analysis and Review of Job Insecurity and its Consequences. Journal of Occupational Health Psychology, 7(3), 242. https://doi.org/10.1037/1076-8998.7.3.242

Valenzuela, H. C. (205). Precariedad, precarización y trabajo precario [Precariousness, Precariousness and Precarious Work]. Polis, 40. Recuperado de https://journals.openedition.org/polis/10754

Vanhercke, D., De Cuyper, N., Peeters, E. y De Witte, H. (2014). Defining Perceived Employability: A Psychological Approach. Personnel Review, 43(4), 592-605. https://doi.org/10.1108/PR-07-20120110

Warr, P. (1990). The Measurement of Well-Being and other Aspects of Mental Health. Journal of Occupational Psychology, 63, 193-210. https://doi.org/10.1111/j.20448325.1990.tb00521.x

\title{
DIFFERENCES BETWEEN EMPLOYABILITY, JOB INSECURITY AND HEALTH IN ORKERS AND UNEMPLOYED
}

\author{
Miguel Angel Serrano Rosa ${ }^{1}$, SANDra BaEnA ${ }^{1} \mathrm{Y}$ \\ FRANCISCO MOLINS-CORREA $^{1}$
}

\section{EXTENDED SUMMARY}

\section{Introduction}

The advancement of new technologies and the globalization of markets has supposed a transition from the traditional forms of capitalism to the so-called "flexible capitalism" that currently prevails (Sennett, 2006). Flexible Capitalism is characterized by its flexibility and lack of stability (Sisto, 2009; Valenzuela, 2014) influencing new labor practices, such as flexibilization, outsourcing, work in temporary teams, subcontracting, etc. (Valenzuela, 2014). These new labor relationships imply a precarious job (Rivas, 2006; Standing, 2011; Valenzuela, 2014), producing job insecurity. This change also places all the responsibility of hiring on the worker, demanding absolute availability, an "adaptive behavior" (Sennett, 2006) and always being the best professional in the best conditions. In this context, the global financial crisis, which began at the end of 2007, brought significant reduction in work, increasing the deterioration of existing employment conditions (Rocha \& Aragón, 2012) and leaving many workers unemployed.

From this complicated context derives, on the one hand, (1) that the current employment situation hinders the reincorporation of unemployed people, leaving them more exposed to psychological risk factors. Furthermore, this is 
associated with the increase or exacerbation of mental illnesses such as anxious-depressive symptoms, somatoform disorders and/or sleep disorders. Moreover, risk behaviors such as the consumption of alcohol, tobacco and other substances, and family conflicts and suicide cases has been described (e.g., Allebeck, 2013; Bernal, Gasparrini, Artundo, \& McKee, 2013; Granado, 2014; Salvador-Carrulla \& Roca, 2013). On the other hand, (2) the job insecurity (fear of losing employment) can cause a loss of job satisfaction in workers (Sverke, Hellgren, \& Näswall, 2002), a feeling of anguish (Valenzuela, 2014) and a stress similar to the loss of work itself, related to poor psychological health.

In this situation, it becomes clear that there is a need to look for strategies that reduce the harmful effects of unemployment and job insecurity. In this setting, the concept of employability should be emphasized. Many authors understand employability as a personal ability to incorporate to the working world, maintain a job or obtain a new one (e.g., Hillage \& Pollard, 1998; Rousseuau, 1997). However, employability can be considered as (Bargsted, 2008): (1) objective employability (Bargsted, 2017) and (2) subjective employability. Vanhercke et al. (2014) states that the subjective measure of beliefs (subjective employability) held by individuals on the ease of finding a new job (De Cuyper et al., 2011a, b, 2014; Kirves et al., 2014). In a deteriorated labor market, where objective employability is diminished (Bargsted, 2017) and where the focus of recruitment is on the individuals, it seems necessary focusing on workers to achieve an increase in their perceived employability (Bargsted, 2017).

In addition to the positive relationship that seems to be found between employability and the possibilities of obtaining or maintaining employment, there is another line of research that understands employability as a buffering variable of the harmful consequences of unemployment and job insecurity. Employability has been related to selfefficacy and affective well-being (McArdle, Waters, Briscoe, \& Hall, 2007), job satisfaction, well-being and general health status (e.g., Bernston \& Marklund, 2007; De Cuyper, Bernhard-Oettel, Bernston, De Witte, \& Alarco, 2008; Gamboa, Gracia, Ripoll, \& Peiró, 2007). Therefore, given the current increase of unemployment and the labor market insecurity produced by new forms of work, it seems interesting to develop employability in both, the unemployed and the working population. Thus, unemployed would benefit from greater potential in the search for employment and reduce the risk of mental illness (McArdle et al., 2007; Vanhercke et al., 2014); and, on the other hand, workers would reduce the harmful effects produced by job insecurity, prevent the loss of their work and, in case of loss, be prepared to face the unemployment situation proactively, increasing their possibilities of reintegration (Bernston \& Marklund, 2007; De Grip, van Loo, \& Sanders, 2004).

Therefore, our study aims to analyze the role of employability as a variable that can influence job insecurity and health at psychological level, in workers and unemployed.

\section{Method}

\section{Participants}

The sample consists of 97 participants (age range: 18 to 62 years old). $48.5 \%$ are men and $51.5 \%$ are women. As far as the labor situation is concerned, the sample is divided into $63.9 \%$ of people who are active -62 working participants-, and $36.1 \%$ unemployed -35 participants-. Of the subset of active people, all worked in the same area of the Valencian community affected by the labor crisis and belonged to an industry in the agricultural sector. It was found that there were no significant differences in age, neither in family or economic charges. This study was conducted in 2015.

\section{Material}

The methodology of this exploratory study was quasiexperimental. Thus, after adapting the questionnaires according to the two subgroups in which the sample is divided -workers and unemployed-, they were distributed among the study participants. In these questionnaires, two types of variables were measured, (1) socio-demographic variables, and (2) trait / situational variables: general selfefficacy, dispositional optimism, job satisfaction, per- 
ceived general health. In addition, as main variables of this study, employability and job insecurity were measured.

\section{Statistical analysis}

To test the hypotheses, first, it was checked if there were differences between the workers and the unemployed in all the previous variables. Subsequently, the median was used in order to split the sample into high and low employability, and high and low job insecurity in the case of active workers.

\section{Results}

\section{Labor situation, employability and psychosocial health}

Unemployed sample have a statistically higher scores on employability than active population. Unemployed also present a significantly higher perceived health scores than working population sample. Finally, optimism presented a marginal effect, with a higher score for the unemployed.

\section{Employability and psychological variables}

In the case of employability, people who score higher in this construct had levels of satisfaction, significantly higher than people with low employability. In addition, the levels of affective discomfort (depression and anxiety parameters) were statistically higher in people with low employability.

\section{Job insecurity and related psychological variables}

In terms of job insecurity, workers who had higher scores in insecurity had significantly lower scores in job satisfaction and self-efficacy. In addition, high insecurity workers have higher scores in depression, anxiety and general health in comparison to low insecurity. Finally, in the working population, the higher employability the lower scores in labor insecurity.

\section{Discussion}

The results obtained must be observed under the prism of a population that has suffered an economic crisis. Thus, in the first place, supporting the initial hypothesis, it is observed that unemployed had greater employability than those who were currently working. This result could be interpreted considering the need of the unemployed to look for strategies to increase the chances of finding a job; that is, unemployed people must dedicate more effort and time to promote their work skills in order to be able to change their employment status and be able to enter the labor market. On the other hand, in the light of this result, we hypothesize that these differences could be maximized if working people do not spend time (or not have time) to promote their employability. On the other hand, contrary to the hypothesis, the unemployed have a higher perception of general health and optimism. This result could be supporting the idea that working has a negative impact on psychosocial health, supporting Granado's (2014) approach regarding the similarity between the harmful effects of working conditions and those of unemployment.

On the other hand, fulfilling our hypothesis, we find that having high employability is related to psychosocial health variables. Specifically, those who have higher scores in this construct have lower scores in anxiety and depression, and higher scores in satisfaction, compared to those with lower employability.

Finally, and focusing on the working sample, we can verify that job insecurity is related to harmful effects such as unemployment itself. Thus, those who perceive their employment as more insecure also showed greater emotional distress and, consequently, lower job satisfaction. One possible explanation could be that a worker would feel helplessness in the case of possible loss of work that, in addition, it is perceived as less self-effective. Finally, we can add more evidence about the relationship that employability has with job insecurity. Thus, those who show greater employability, have lower scores in labor insecurity (Sverke et al., 2002; Valenzuela, 2014).

The implications of these results could be, in the first place, the need to check the hypothesis of whether the active population is lacking in employability and therefore it 
should be promoted. The second implication is that having high or low employability or insecurity influences the level of psychological distress, satisfaction and optimism. That is, promoting employability and reducing insecurity would reduce the likelihood of changes in mood and therefore positively influence psychosocial health. Therefore, within a preventive approach the promotion of employability would allow workers with more skills to find or maintain a job as well as having better emotional health.

\section{Conclusions}

This study highlights the differences in employability and health between workers and unemployed in the context of flexible capitalism. As well our results emphasize the importance of employability as a key factor, in workers and unemployed, not only because of its facilitating role in obtaining or maintaining employment, but also for its beneficial effects on health. On the other hand, the perception of job insecurity is also presented as a key aspect when interpreting the emotional state of workers that could be modulated by fostering employability. In conclusion, in the light of these results, we believe that the promotion of employability is necessary for obtaining or maintaining an employment and having better emotional health. 
